\title{
The relationship between muscle mitochondrial nutritional overloading and insulin resistance
}

\author{
Jae-Han Jeon ${ }^{1}$, Jun-Sung Moon ${ }^{2}$, Kyu-Chang Won ${ }^{2}$, In-Kyu Lee ${ }^{1}$ \\ ${ }^{I}$ Department of Internal Medicine, Kyungpook National University School of Medicine; ${ }^{2}$ Department of Internal Medicine, \\ Yeungnam University College of Medicine, Daegu, Korea
}

The incidence of type 2 diabetes mellitus and insulin resistance is growing rapidly. Multiple organs including the liver, skeletal muscle and adipose tissue control insulin sensitivity coordinately, but the mechanism of skeletal muscle insulin resistance has not yet been fully elucidated. However, there is a growing body of evidence that lipotoxicity induced by mitochondrial dysfunction in skeletal muscle is an important mediator of insulin resistance. However, some recent findings suggest that skeletal mitochondrial dysfunction generated by genetic manipulation is not always correlated with insulin resistance in animal models. A high fat diet can provoke insulin resistance despite a coordinate increase in skeletal muscle mitochondria, which implies that mitochondrial dysfunction is not mandatory in insulin resistance. Furthermore, incomplete fatty acid oxidation by excessive nutrition supply compared to mitochondrial demand can induce insulin resistance without preceding impairment of mitochondrial function. Taken together we suggested that skeletal muscle mitochondrial overloading, not mitochondrial dysfunction, plays a pivotal role in insulin resistance.

Keywords: Insulin resistance; Mitochondria; Lipotoxicity; Fatty acid oxidation; Reactive oxygen species

\section{INTRODUCTION}

The incidence of type 2 diabetes mellitus (DM), metabolic syndrome and obesity has been rapidly increasing over the last few decades. The pathogenesis of type $2 \mathrm{DM}$ is mainly characterized by pancreatic beta cell dysfunction and peripheral insulin resistance. Although the mechanism of insulin resistance, which is defined by the condition in which normal amounts of insulin are inadequate to produce a normal insulin response from fat, muscle and liver cells, has yet to be fully

Received: April 21, 2017, Revised: May 30, 2017

Accepted: June 7, 2017

Corresponding Author: In Kyu Lee, Division of Endocrinology and Metabolism, Department of Internal Medicine, Kyungpook National University School of Medicine, 130

Dongdeok-ro, Jung-gu, Daegu 41944, Korea

Tel: +82-53-420-5564, Fax: +82-53-420-2046

E-mail: leei@knu.ac.kr elucidated, recent studies have offered some insight into the role of skeletal muscle insulin resistance in type $2 \mathrm{DM}$.

Skeletal muscle plays a major role in insulin-mediated glucose uptake, and works as a major reservoir for glucose storage. In the insulin resistant state, impaired insulin-stimulated glucose uptake and reduced glycogen synthesis accounts for more than $50 \%$ of the reduction in whole body glucose disposal [1]. Furthermore, abnormal fatty acid metabolism plays a role in insulin resistance. Excess fat deposition in non-adipose tissue (liver, muscle, pancreatic beta cells) in type $2 \mathrm{DM}$ can be induced by multiple mechanisms such as nutrient excess, which is quite common in insulin resistant individuals, accelerated lipolysis in adipose tissue by insulin resistance, or reduced fatty acid (FA) oxidation (FAO) in mitochondrial defect. Moreover, this increased lipid content, especially in muscle, is linked with insulin resistance [2,3]. As skeletal mitochondrial deficiency was observed in patients with insulin resistance or type $2 \mathrm{DM}$, it has been proposed as a key player

Copyright (C) 2017 Yeungnam University College of Medicine

This is an Open Access article distributed under the terms of the Creative Commons Attribution Non-Commercial License (http://creativecommons.org/licenses/by-nc/4.0/) which permits unrestricted non-commercial use, distribution, and reproduction in any medium, provided the original work is properly cited. 
in skeletal muscle lipid accumulation [4]. Here, we summarize the causal role of skeletal muscle mitochondria in mediating insulin resistance, with a focus on some recent evidence that mitochondrial dysfunction does not precede insulin resistance.

\section{Molecular mechanism of skeletal muscle insulin resistance: with a focus on links between skeletal muscle mitochondrial dysfunction and lipotoxicity}

Tissue-specific insulin sensitivity was first found to decrease in type $2 \mathrm{DM}$ in 1940 [5]. In 1975, diminished ability of insulin to promote glucose uptake in type 2 DM was demonstrated [6]. A following study by DeFronzo revealed markedly decreased whole body glucose disposal during insulin clamp in type $2 \mathrm{DM}$ [7]. As skeletal muscle is a major reservoir for postprandial glucose storage, which accounts for $80 \%$ of insulin-stimulated glucose disposal [8], the mechanism of skeletal muscle insulin resistance has been thoroughly investigated. NADH-O2 oxidoreductase and citrate synthase (CS) activity in skeletal muscle of type $2 \mathrm{DM}$ were shown to be decreased, and a concurrent decrease in the size of mitochondria was observed upon electron microscopy [9]. In patients with type $2 \mathrm{DM}$ or prediabetes, decreased expression of oxidative phosphorylation (OXPHOS) genes as well as NRF-1 and proliferator-activated receptor gamma coactivator 1 (PGC-1) $\alpha / \beta$ genes, the master regulators of mitochondrial biogenesis, was observed [10]. A decrease of genes involved in OXPHOS, which was induced by $P G C-1 \alpha$, was observed in a subsequent study [11]. Indeed, this decrease was observed not only in subjects with type $2 \mathrm{DM}$, but also in their lean insulin-resistant offspring, who showed a $30 \%$ reduction in mitochondrial OXPHOS based on ${ }^{31} \mathrm{P}$-magnetic resonance spectroscopy $\left({ }^{31} \mathrm{P}-\mathrm{MRS}\right)$. Furthermore, this was accompanied by an $80 \%$ increase in intramyocellular lipid (IMCL) content [12]. Similarly, the same authors found that mitochondrial density was reduced by $38 \%$ and insulin receptor substrate-1 (IRS-1) serine phosphorylation was increased in the muscle of insulinresistant offspring [13]. Lipid intermediates such as long chain fatty acyl coenzyme A (CoA), diacylglycerols, and ceramides in skeletal muscle are regarded as important regulators of insulin resistance [14]. These intermediates can activate serine/ threonine kinases such as protein kinase $\mathrm{C}$ in skeletal muscles
[15], which then activate phosphorylates serine residues in IRS-1, thereby inhibiting insulin-induced phosphatidylinositol 3-kinase activity. Taken together, mitochondrial deficiency and fat oxidation and the subsequent increase of intramyocellular triglyceride was proposed as a key mediator of insulin resistance [4]. It has also been hypothesized that reduced primary mitochondrial function predisposes one to insulin resistance [13]. Though seemingly very robust and conclusive, questions still arise as to whether increased IMCL is the direct effect of mitochondrial dysfunction and whether IMCL acts as a deleterious factor in insulin sensitivity. Skeletal muscle of trained endurance athletes is markedly insulin sensitive and has a high oxidative capacity, despite having an elevated lipid content [16], and their OXPHOS capacities are not disturbed despite the high IMCL content [17]. These findings suggest that an increased IMCL level is not sufficient to cause mitochondrial damage or insulin resistance. Furthermore, careful consideration is needed when determining whether increased IMCL is the consequence of mitochondrial deficiency/dysfunction or merely derived from increased fat supply, because the possibility that mitochondrial dysfunction is secondary to insulin resistance still resides in this phenomenon.

The latter theory seems to be quite attractive in terms of mitochondrial bioenergetics. The rate of FAO or mitochondrial respiration is not dependent on the rate of fat supply, but rather on energy demand, which is comprised of idling activity plus energy required for adenosine triphosphate (ATP) generation. In other words, under resting condition, when demand for ATP synthesis is decreased, the basal idling activity for protein synthesis, maintenance of membrane potential and proton leaks accounts for most of the mitochondrial energy demand. Skeletal muscle, in general, harbors enormous mitochondrial reserve and can flexibly increase its substrate flux and ATP synthesis according to increases in exercise capacity. Boushel et al. found that skeletal muscle mitochondrial function was normal in type $2 \mathrm{DM}$, only its number was deficient [18]. As stated above, a 30\% reduction in mitochondrial number and OXPHOS capacity is observed in skeletal muscle of type 2 DM. Since mitochondria can flexibly increase its oxidative capacity up to several times during exercise (up to 150 times in very healthy, trained individuals), it can overcome $30 \%$ loss of mitochondria or OXPHOS capacity [19]. These findings can also indicate that subtle defects in OXPHOS have little impact on IMCL accumulation because OXPHOS 
almost never works at maximal capacity during usual activities. Moreover, as substrate oxidation rate is not dependent on supply, but rather on mitochondrial demand, lipids can accumulate in skeletal muscle when the supply exceeds demand, regardless of its mitochondrial OXPHOS capacity [20].

Collectively, these conflicting results have shown the possibility that insulin resistance might not be governed by skeletal mitochondrial dysfunctions. The following studies introduced here provide some evidence that skeletal muscle mitochondrial dysfunction is not a prerequisite to the development of insulin resistance.

\section{Relationship between mitochondrial function and insulin resistance: preclinical results}

A previous in vitro study designed to investigate impaired cellular respiratory function by treatment with the mitochondrial inhibitor sodium azide showed, on the contrary to expectations, increased basal glucose uptake, phosphorylation of AMP-activated protein kinase (AMPK) and GLUT4 mRNA expression [21]. Gene manipulation studies in animals have also shown some evidence indicating that skeletal muscle mitochondrial dysfunction does not correlate with insulin resistance. A mouse model with respiratory chain dysfunction in skeletal muscle generated by muscle-specifically knocking-out mitochondrial transcription factor A showed paradoxically increased peripheral glucose disposal during a glucose tolerance test, indicating that mitochondrial dysfunction in skeletal muscle might not be a primary etiological event in insulin resistance [22]. The skeletal muscle specific ablation of PGC-1 $\alpha$ in mouse showed that PGC- $1 \alpha$ was necessary for normal regulation of mitochondrial gene expression. However, a reduction of PGC- $1 \alpha$ levels in skeletal muscle and the subsequent reductions in OXPHOS gene expression were not causally linked to systemic insulin resistance, although of the possibility that PGC- $1 \alpha$ and OXPHOS genes contribute to insulin resistance in muscle under other experimental conditions cannot be ruled out [23]. Conditional deletion of apoptosis inhibiting factor (AIF) is known to induce progressive loss of respiratory chain and OXPHOS dysfunction. Muscle- and liver-specific AIF ablation in mice expressing a pattern of OXPHOS were thus generated to look for the relationship between mitochondrial deficiency and insulin resistance. The intervention resulted in paradoxically reduced fat mass and ameliorated insulin sensitivity [24].

Recently, it was shown that heterozygous Crif1 deletion in the skeletal muscle was not associated with insulin resistance. Crif1 is a mitochondrial protein required for the intramitochondrial production of mtDNA encoded OXPHOS subunits, and targeted elimination of the Crif1 gene causes a phenotype that shows organ-specific failure of OXPHOS. These findings supported the hypothesis that skeletal muscle mitochondrial deficiency does not mediate insulin resistance [25]. Collectively, although these gene manipulation studies revealed rather universal results, they require attention during interpretation because they casually reflect extreme cases that might lead to the activation of other compensatory mechanisms. Indeed, in some studies, AMPK level was increased or showed a tendency to increase, which could in turn increase insulin sensitivity, mimicking a "metformin effect." To increase AMPK, ATP content is supposed to be sufficiently inhibited by intervention. However, this phenomenon is less likely to be reproduced in in vivo studies of human or animals with insulin resistance.

\section{Relationship between mitochondrial dysfunction and insulin resistance in human type 2 diabetes}

Several lines of human studies have also shown that skeletal mitochondrial dysfunction does not act as a direct cause of insulin resistance. In a recent human study, mitochondrial function was measured among three different groups: type 2 DM patients, subjects with impaired fasting glucose, impaired glucose tolerance and/or recently diagnosed type 2 $\mathrm{DM}$, and healthy, normoglycemic controls. In vivo mitochondrial function measured by ${ }^{31} \mathrm{P}-\mathrm{MRS}$ showed no differences in phosphocreatinine (PCr) and adenosine diphosphate recovery time and maximum aerobic capacity between groups. These findings suggest that mitochondrial dysfunction does not necessarily manifest either cause or consequence of insulin resistance [26]. Another human study showed that, in patients with type $2 \mathrm{DM}$, mitochondrial respiration was lower than in healthy controls. However, when mitochondrial respiration was normalized for mitochondrial DNA content 
or CS activity, there were no differences in OXPHOS or electron transport capacity between patients with type $2 \mathrm{DM}$ and healthy control subjects. The authors concluded that blunting of coupled and uncoupled respiration in type 2 diabetic patients is not attributed to mitochondrial dysfunction, but rather to lower mitochondrial content. It is well known that exercise training increases mitochondrial content in skeletal muscle and aging diminishes mitochondrial OXPHOS capacity; thus, it can be hypothesized that this decreased mitochondrial content may be attributed to sedentary life style and old age in patients with T2DM [18]. Asian Indians are known to have a higher susceptibility to diabetes than Europeans. Thirteen diabetic Indians, 13 nondiabetic Indians, and 13 nondiabetic Northern European Americans were compared. Despite being more insulin resistant, diabetic Indians had similar muscle OXPHOS capacity as nondiabetic Indians, suggesting that diabetes per se does not cause mitochondrial dysfunction. Regardless of their diabetic status, Indians had higher OXPHOS capacity than Northern European Americans, although they were substantially more insulin resistant, indicating a dissociation between mitochondrial dysfunction and insulin resistance [27].

The hypothesis that mitochondrial dysfunction acts as a consequence rather than a cause of insulin resistance is further supported by a study showing that, when plasma insulin and glucose concentrations were maintained at similar postabsorptive levels, skeletal muscle ATP production and mtDNA copy numbers were similar in both type 2 diabetic and nondiabetic groups, suggesting that reduced muscle mitochondrial content is not a universal finding in type 2 diabetic patients [28].

\section{The role of high-fat diet in uncoupling of mitochondrial dysfunction from insulin resistance}

C57BL/6J mice fed a short-term high fat diet showed altered expression levels of genes involved in a variety of biological processes, including energy metabolism, lipogenesis, and immune function, as well as increased levels of complexes I, II, III, IV, and V of OXPHOS, despite insulin resistance [29]. Another mouse model of free fatty acid challenge showed insulin resistance despite an increase in muscle mitochondria.
In this study, activation of peroxisome proliferator-activated receptor (PPAR) $\delta$ by fatty acids subsequently increased PGC$1 \alpha$ protein expression in posttranscriptional manner, thereby increasing mitochondrial biogenesis. Mitochondrial DNA copy numbers and mitochondrial enzyme proteins such as CS and cyclooxygenase 1 (COX1) were increased [30]. Additionally, C57BL/6J mice treated with a high fat diet displayed elevated palmitate oxidation rate and palmitoyl-CoA oxidation in isolated mitochondria compared with chow diet controls. Highfat diet groups exhibited significant elevation of PGC- $1 \alpha$ and mitochondrial respiratory chain subunits, despite impaired glucose tolerance relative to controls [31]. Rats fed a high-fat diet and given daily injections of heparin to increase free fatty acid levels showed an increase in mitochondrial biogenesis in muscle [32].

An interventional study of one-week treatment of acipimox to lower plasma fatty acid levels paradoxically decreased the PGC-1- and nuclear-encoded mitochondrial genes, despite increasing the glucose disposal rate and insulin sensitivity [33]. Taken together, these results contradict with the preexisting paradigm that reduced FAO mediates lipid-induced insulin resistance. More direct evidence that mitochondrial deficiency is not an etiological event but rather a consequence of insulin resistance was obtained from a study conducted by Bonnard et al. [34]. Mice fed a high-fat, high-sucrose diet (HFHSD) were shown to be glucose intolerant after 4 weeks, but their mitochondrial function was normal. After 16 weeks, these mice were diabetic, and mitochondrial dysfunction occurred, as demonstrated by reduced mitochondrial density and altered structure observed upon electron microscopy, as well as reduced mitochondrial gene expression (Cox1, Cox3, PGC-1 $1 \alpha$ ). The results of this study indicated that insulin resistance could be induced by excessive nutrition without preceding mitochondrial dysfunction, and rather, the mitochondria plays as a victim.

\section{Skeletal muscle fat oxidation in insulin resistant humans}

If mitochondrial dysfunction is a key player in insulin resistance, the fat oxidation rate should be diminished in insulin resistant or obese subjects. Although the majority of previous studies support this hypothesis, some studies show the oppo- 
site results. A previous study of fat metabolism in long-term diagnosed type 2 DM revealed that basal whole body fat oxidation rates were significantly increased compared to controls [35]. Another study showed that mitochondrial function is normal and fat oxidation capacity is increased in both leg and arm muscles in obese humans [36]. Holloway et al. demonstrated that palmitate oxidation was not decreased in skeletal muscle of obese women [37]. Moreover, in a subsequent review, they concluded that obesity itself does not alter the ability of skeletal muscle mitochondria to oxidize fatty acids, and that obesity-related reductions in skeletal muscle $\mathrm{FAO}$ can be attributed to reductions in mitochondrial content and not to intrinsic alterations or dysfunction within the mitochondria [38]. Taken together, these findings suggest that mitochondrial dysfunction is not associated with insulin sensitivity.

\section{Incomplete fatty acid oxidation as a cause of insulin resistance}

Although a majority of previous reports support the idea that skeletal muscle mitochondrial function mediates insulin resistance, recent findings from animal studies showing that high fat diet induces insulin resistance without exacerbating mitochondrial function or even coordinately increasing mitochondrial biogenesis have made this interpretation puzzling. Thus, the complex relationships between the degree of skeletal muscle FAO and insulin resistance have been investigated. PPAR- $\alpha$ and PPAR- $\beta / \delta$ serve as controllers of the cellular lipid metabolic pathway [39]. Transgenic mice overexpressing PPAR $\alpha$ in a muscle specific manner (MCK-PPAR $\alpha$ ) showed increased beta oxidation and protection from diet-induced obesity compared to controls, but surprisingly, they developed insulin resistance [40]. Conversely, PPARk $\alpha$ nock out $(\mathrm{KO})$ mice were protected from diet-insulin resistance [41]. Interestingly, in this study, AMPK activity was diminished in MCK-PPAR $\alpha$, and AMPK activator 5-aminoimidazole4-carboxamide ribonucleotide treatment reversed the negative effect of PPAR $\alpha$ on glucose transporter (GLUT) 4 expression [41]. This finding led to the possibility that alterations in cellular energetics were involved in glucose intolerant models. Dinitrophenol, a protonophore that uncouples mitochondrial electron transport from OXPHOS, was found to reverse the inhibitory effects of PPAR $\alpha$ on expression of GLUT4 and acetyl-CoA carboxylase $\alpha$ (ACC) phosphorylation. Additio- nally, skeletal muscle FAO inhibition by oxfenicine improved glucose tolerance in a MCK-PPAR $\alpha$ model, despite exacerbated skeletal muscle lipid accumulation. Taken together, these findings have led to revisiting of the Randle effect, which first described the reciprocal allosteric inhibition of glucose metabolism by high-level fatty acid utilization [42].

Inhibition of malonyl-CoA decarboxylase (MCD) results in partial carnitine palmitoyltransferase 1 inhibition. Decreased $\beta$-oxidation by MCD KO resulted in protection from insulin resistance after a 12-week high fat diet in mouse models [43]. Conversely, chronic high-fat feeding has been shown to increase the effects of postprandial serum non-esterified fatty acids (NEFAs) and acylcarnitines in serum, and this phenomenon was related insulin resistant states. Even-chain acylcarnitines (C6-C22), which represent incomplete $\beta$-oxidation, were increased. This model was marked by increased whole body fat oxidation, impaired switching to carbohydrate substrate during the fasted to fed transition, and coincident reductions in muscle levels of several TCA cycle intermediates. Collectively, the results have shown that obesity, diabetes, and HF feeding are accompanied by increased rather than decreased rates of $\beta$-oxidation in skeletal muscle and suppression of mitochondrial fatty acid importation protects against lipid-induced insulin resistance [43].

Chronic high fat feeding can lead to adaptive response in muscle shown as an increase of enzymes involved in FAO; however, this adaptation is not accompanied by parallel increases in enzymes of the TCA cycle. The result of this imbalance is the accumulation of incompletely oxidized metabolites such as acylcarnitines. A more recent study compared plasma concentrations of carnitine and acylcarnitines between fasting, obese nondiabetics and patients with type $2 \mathrm{DM}$ and found that the total summed acylcarnitine concentration and medium-chain acylcarnitines, especially acetylcarnitine levels, were increased in diabetics [44]. Additionally, a positive correlation between acetylcarnitine and glycated hemoglobin level was observed. Moreover, in this study, acylcarnitines activated NFKB in a RAW264.7 cell model, which was previously suggested to promote serine phosphorylation of IRS-1 and thereby play a role in insulin resistance [45]. The authors have hypothesized that reduced mitochondrial number, volume, and mitochondrial substrate oxidation capacity in muscles of type 2 DM might trigger limitation of tricarboxylic acid (TCA) utilization, thereby contributing to inefficient, in- 
complete LCFA $\beta$-oxidation. However, careful interpretation is required because this was an observational study; therefore, not conclusions regarding whether decreased mitochondrial content is a primary event or secondary to insulin resistance could be made. Taken together, although seemingly conflicting, incomplete FAO arises from an imbalance between increased fatty acid import and/or decreased TCA cycle capacity, and this relatively excessive FAO compared to demand of TCA cycle results in insulin resistance via activation of $\mathrm{NFKB}$.

\section{ROS as a mediator of skeletal mitochondrial dysfunction and insulin resistance}

In a recent study, insulin resistance was induced by treatment of TNF- $\alpha$ or dexamethasone in mice. These mice were then treated with two small molecules and four transgenes, each of which were designed to lower ROS levels, and therefore ameliorated insulin resistance [46]. The following study by Bonnard et al. showed that 16 weeks of chronic high sucrose high fat diet (HSHFD) increases protein carbonylation, which is a marker of oxidative stress, whereas at 4 weeks, carbonylation level is not altered. On the other hand, insulin resistance was already observed after providing a HSHFD for 4 weeks [34]. In the same study, streptozotocin-induced mice showed decreased mitochondrial DNA content; however, this was recovered by antioxidant $\mathrm{N}$-acetylcysteine treatment, again suggesting that mitochondrial deficiency is not a primary phenomenon, but rather a response of increased mitochondrial oxidative stress. The following study has suggested that mitochondrial hydrogen peroxide $\left(\mathrm{H}_{2} \mathrm{O}_{2}\right)$ emissions serve as a link between excess fat intake and insulin resistance [47]. High-fat diet increases mito- chondrial $\mathrm{H}_{2} \mathrm{O}_{2}$-emitting potential, while this is prevented by the antioxidant SS31. To specifically determine whether mitochondrial $\mathrm{H}_{2} \mathrm{O}_{2}$ emissions are a primary factor in the development of diet-induced insulin resistance, transgenic C57BL/6J mice overexpressing human catalase targeted specifically to mitochondria in skeletal and cardiac muscle were evaluated. Scavenging of $\mathrm{H}_{2} \mathrm{O}_{2}$ by the malonyl CoA-acyl carrier protein transacylase prevented high fat diet induced insulin resistance, which was confirmed by an ameliorated HOMA index and oral glucose challenge test [52]. Mitochondrial function is known to be reduced by the aging process, ultimately leading to muscle insulin resistance.
To verify the role of ROS in this process, a mouse model of overexpression of the human catalase gene to mitochondria (MCAT) was designed. In old wild type mice, the p-AMPK/ AMPK ratio, p-ACC2 and PGC1 $\alpha$ protein levels and mitochondrial density were decreased, and these decreases were recovered in old MCAT mice. The authors hypothesized that mitochondria-generated ROS production decreases AMPK, thereby decreasing mitochondrial biogenesis, resulting in less fat oxidation and finally insulin resistance [48]. More recently, NEFA and insulin treatment in rats were shown to trigger insulin resistance, which was associated with high mitochondrial ROS generation and activation of the NFKB pathway [49]. The ratio of oxidized glutathione (GSSG) to reduced glutathione (GSH) and the superoxide anion production rate were increased. Mitochondrial enzyme activity and ATP production were not impaired by changes in ROS generation under any condition. These findings suggested that skeletal muscle mitochondria plays a small role, at least in acute changes in ROS production rate or insulin resistance caused by high fat diet. Collectively, these studies suggest that ROS is a key mediator of insulin resistance; however, the role of mitochondrial dysfunction is still puzzling. Some studies have indicated that changes in mitochondrial function are negligible in ROS-mediated insulin resistance models [49], while the others showed somewhat substantial decreases in mitochondrial function in subjects with insulin resistance. FisherWellman et al. recently proposed the role of mitochondrial $\mathrm{H}_{2} \mathrm{O}_{2}$ emission as the primary cause of IR [50]. The main redox buffering system, glutathione (2GSHG/GSSG) and thioredoxin, which are known as "sulfur switches," derive their reducing power from nicotin amide adenine dinucleotide phosphate and thereby mitigate oxidative stress. However, when mitochondrial supply exceeds demand in response to the chronic high fat diet, mitochondrial $\mathrm{H}_{2} \mathrm{O}_{2}$ emissions increase, reducing reserve capacity within the redox-buffering system and inducing an oxidative shift in the cellular redox environment. In general, phosphatase activity is decreased during oxidation of redox environments; therefore, stress-sensitive kinases such as c-Jun $\mathrm{N}$-terminal kinase, extracellular signal - regulated kinases, and inhibitors of $\mathrm{NFkB}$ can lead to the subsequent Ser/Thr phosphorylation of IRS1, ultimately limiting insulin sensitivity. Taken together, these findings indicate that it is likely that chronic nutrient excess induces mitochondrial overload and increases mitochondrial ROS pro- 
duction, and this oxidized mitochondrial environment can disrupt insulin signaling. The mitochondrial dysfunction itself seems to act more as a result of this process than a cause.

\section{CONCLUSION}

In recent years, the mechanism of insulin resistance has
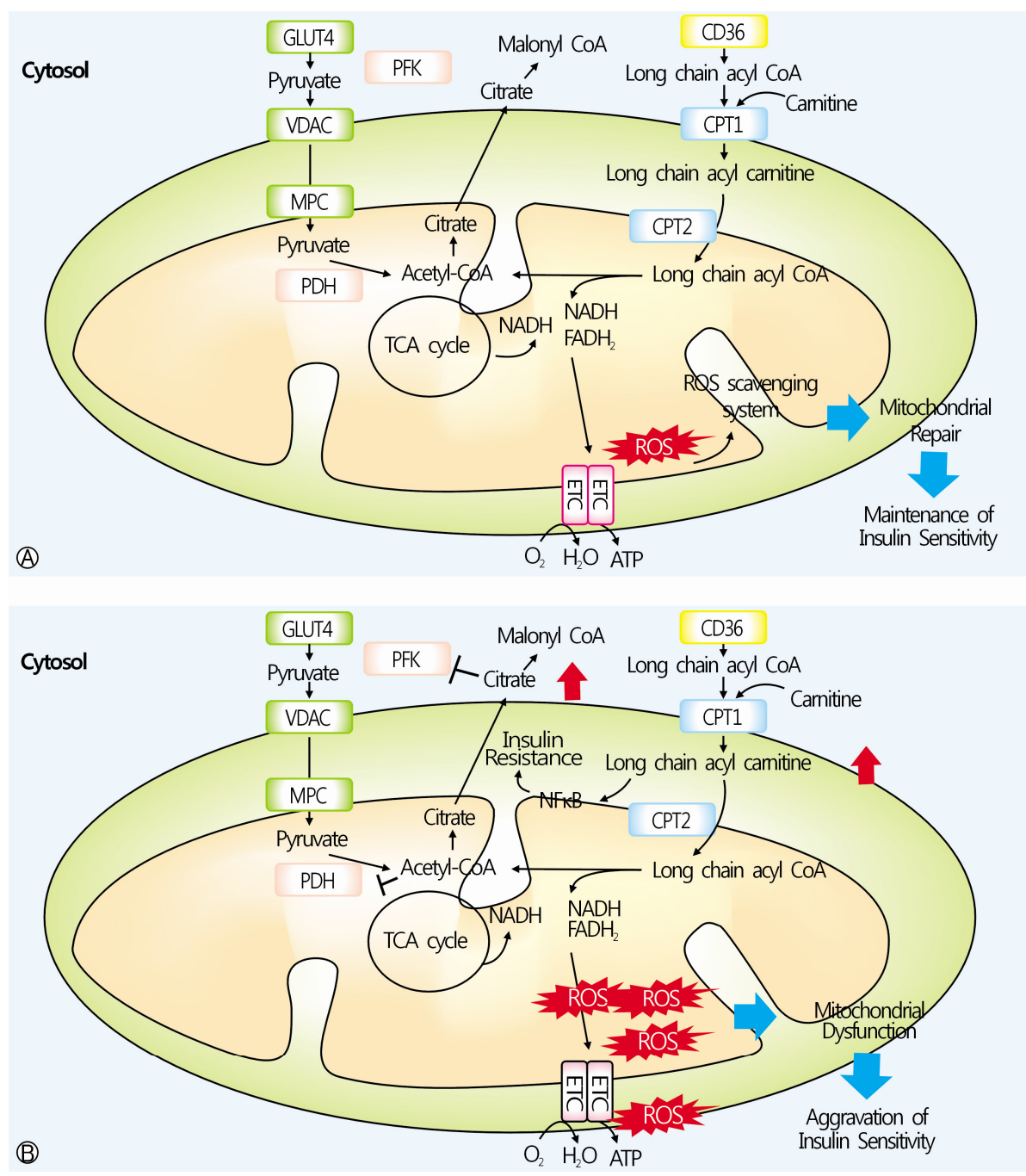

Fig. 1. The impact of mitochondrial nutritional overloading in mitochondrial function and insulin resistance in the skeletal muscle. (A) Under nutritionally homeostatic condition, ROS produced by OXPHOS is readily scavenged by redox buffering system, which maintains mitochondrial function. At the same time, the byproduct of incomplete fatty oxidation such as acylcarnitine is hardly detected. (B) Under nutritionally excess status, especially when FFA influx exceeds mitochondrial capacity to oxidize it, incomplete fatty oxidation occurs, which elicits acylcarnitine abundance. This, in turn, stimulates profinflammtory cytokines such as nuclear factor kappa B which plays a major role in insulin resistance. At the same time, acetyl CoA and citrate derived from exogenous FFA inhibits PDH and PFK, respectively, both of which are critical regulator of glucose uptake and oxidation in the muscle. During OXPHOS, a larger amount of ROS is synthesized which surpasses the capacity of anti-oxidant system. This eventually causes mitochondrial dysfunction that further contributes to insulin resistance. ROS, reactive oxygen species; OXPHOS, oxidative phosphorylation; FFA, free fatty acid; PDH, pyruvate dehydrogenase; PFK, phosphofructokinse; MPC, mitochondrial pyruvate carrier; CD36, cluster of differentiation 36; CPT, carnitine palmitoyltransferase; ETC, electron transport chain; GLUT4, glucose transporter type 4; VDAC, voltage-dependent anion channel. 
been widely investigated, and it is rather clear that skeletal muscle mitochondrial deficiency is observed in subjects with insulin resistance or type $2 \mathrm{DM}$. Although the hypothesis that diminished fat oxidation rate contributes to lipotoxicity has gained wide acceptance, there is also evidence that fat oxidation rate is normal or increased in type $2 \mathrm{DM}$, and pharmacologic intervention or genetic manipulation that lowers mitochondrial function paradoxically ameliorates insulin resistance. Additionally, incomplete fatty oxidation and mitochondrial redox biology have recently received attention as an attractive mechanism to explain lipotoxicity induced insulin resistance, which supports the notion that mitochondrial dysfunction is not an initiating event (Fig. 1).

Elucidation of the puzzling relationship between skeletal muscle mitochondria and insulin resistance has been challenging because a long asymptomatic period of insulin resistance precedes overt diabetes; therefore, long term longitudinal studies are required to examine such individuals. The mitochondria a dynamic organelle, and its function is affected by various factors such as aging, insulin resistance, sedentary lifestyle, and oxidative stress. These factors are interrelated, making the interpretation more challenging.

Nevertheless, the importance of the mechanism of skeletal muscle insulin resistance should be emphasized, as it may provide promising insight into future therapeutic potential in the treatment of type $2 \mathrm{DM}$.

\section{ACKNOWLEDGMENTS}

This research was supported by Kyungpook National University Bokhyeon Research Fund, 2015.

\section{CONFLICT OF INTEREST}

The authors have no potential conflict of interest to declare.

\section{ORCID}

In-Kyu Lee, https://orcid.org/0000-0002-2261-7269

Jae-Han Jeon, https://orcid.org/0000-0002-9217-968X

\section{REFERENCES}

1. Shulman GI, Rothman DL, Jue T, Stein P, DeFronzo RA,
Shulman RG. Quantitation of muscle glycogen synthesis in normal subjects and subjects with non-insulin-dependent diabetes by 13C nuclear magnetic resonance spectroscopy. $\mathrm{N}$ Engl J Med 1990;322:223-8.

2. Bays H, Mandarino L, DeFronzo RA. Role of the adipocyte, free fatty acids, and ectopic fat in pathogenesis of type 2 diabetes mellitus: peroxisomal proliferator-activated receptor agonists provide a rational therapeutic approach. J Clin Endocrinol Metab 2004;89:463-78.

3. Groop LC, Bonadonna RC, Shank M, Petrides AS, DeFronzo RA. Role of free fatty acids and insulin in determining free fatty acid and lipid oxidation in man. J Clin Invest 1991;87: 83-9.

4. Morino K, Petersen KF, Shulman GI. Molecular mechanisms of insulin resistance in humans and their potential links with mitochondrial dysfunction. Diabetes 2006;55(Suppl 2):S9-15.

5. Himsworth HP. Insulin deficiency and insulin inefficiency. Br Med J 1940;1(4139):719-22.

6. Ginsberg H, Kimmerling G, Olefsky JM, Reaven GM. Demonstration of insulin resistance in untreated adult onset diabetic subjects with fasting hyperglycemia. J Clin Invest 1975; 55:454-61.

7. DeFronzo RA. Lilly lecture 1987 . The triumvirate: beta-cell, muscle, liver. A collusion responsible for NIDDM. Diabetes 1988;37:667-87.

8. DeFronzo RA, Gunnarsson R, Björkman O, Olsson M, Wahren J. Effects of insulin on peripheral and splanchnic glucose metabolism in noninsulin-dependent (type II) diabetes mellitus. J Clin Invest 1985;76:149-55.

9. Kelley DE, He J, Menshikova EV, Ritov VB. Dysfunction of mitochondria in human skeletal muscle in type 2 diabetes. Diabetes 2002;51:2944-50.

10. Patti ME, Butte AJ, Crunkhorn S, Cusi K, Berria R, Kashyap $\mathrm{S}$, et al. Coordinated reduction of genes of oxidative metabolism in humans with insulin resistance and diabetes: potential role of PGC1 and NRF1. Proc Natl Acad Sci U S A 2003;100: 8466-71.

11. Mootha VK, Lindgren CM, Eriksson KF, Subramanian A, Sihag S, Lehar J, et al. PGC-1alpha-responsive genes involved in oxidative phosphorylation are coordinately downregulated in human diabetes. Nat Genet 2003;34:267-73.

12. Petersen KF, Dufour S, Befroy D, Garcia R, Shulman GI. Impaired mitochondrial activity in the insulin-resistant offspring of patients with type 2 diabetes. N Engl J Med 2004; 350:664-71.

13. Morino K, Petersen KF, Dufour S, Befroy D, Frattini J, Shatzkes N, et al. Reduced mitochondrial density and increased IRS-1 serine phosphorylation in muscle of insulin-resistant offspring of type 2 diabetic parents. J Clin Invest 2005;115: 3587-93.

14. Schmitz-Peiffer C, Craig DL, Biden TJ. Ceramide generation is sufficient to account for the inhibition of the insulin-stimulated PKB pathway in $\mathrm{C} 2 \mathrm{C} 12$ skeletal muscle cells pretreated with palmitate. J Biol Chem 1999;274(34):24202-10.

15. Itani SI, Ruderman NB, Schmieder F, Boden G. Lipid-induced insulin resistance in human muscle is associated with changes 
in diacylglycerol, protein kinase C, and IkappaB-alpha. Diabetes 2002;51:2005-11.

16. Goodpaster BH, He J, Watkins S, Kelley DE. Skeletal muscle lipid content and insulin resistance: evidence for a paradox in endurance-trained athletes. J Clin Endocrinol Metab 2001; 86:5755-61.

17. Befroy DE, Petersen KF, Dufour S, Mason GF, Rothman DL, Shulman GI. Increased substrate oxidation and mitochondrial uncoupling in skeletal muscle of endurance-trained individuals. Proc Natl Acad Sci U S A 2008;105(43):16701-6.

18. Boushel R, Gnaiger E, Schjerling P, Skovbro M, Kraunsøe $\mathrm{R}$, Dela F. Patients with type 2 diabetes have normal mitochondrial function in skeletal muscle. Diabetologia 2007; 50:790-6.

19. Holloszy JO. Skeletal muscle "mitochondrial deficiency" does not mediate insulin resistance. Am J Clin Nutr 2009;89: 463S-6S.

20. Muoio DM, Neufer PD. Lipid-induced mitochondrial stress and insulin action in muscle. Cell Metab 2012;15:595-605.

21. Brown AE, Elstner M, Yeaman SJ, Turnbull DM, Walker M. Does impaired mitochondrial function affect insulin signaling and action in cultured human skeletal muscle cells? Am J Physiol Endocrinol Metab 2008;294:E97-102.

22. Wredenberg A, Freyer C, Sandström ME, Katz A, Wibom $\mathrm{R}$, Westerblad H, et al. Respiratory chain dysfunction in skeletal muscle does not cause insulin resistance. Biochem Biophys Res Commun 2006;350:202-7.

23. Handschin C, Choi CS, Chin S, Kim S, Kawamori D, Kurpad AJ, et al. Abnormal glucose homeostasis in skeletal muscle-specific PGC-1alpha knockout mice reveals skeletal muscle-pancreatic beta cell crosstalk. J Clin Invest 2007;117:3463-74.

24. Pospisilik JA, Knauf C, Joza N, Benit P, Orthofer M, Cani PD, et al. Targeted deletion of AIF decreases mitochondrial oxidative phosphorylation and protects from obesity and diabetes. Cell 2007;131:476-91.

25. Ryu MJ, Kim SJ, Kim YK, Choi MJ, Tadi S, Lee MH, et al. Crif1 deficiency reduces adipose OXPHOS capacity and triggers inflammation and insulin resistance in mice. PLoS Genet 2013;9:e1003356.

26. De Feyter HM, van den Broek NM, Praet SF, Nicolay K, van Loon LJ, Prompers JJ. Early or advanced stage type 2 diabetes is not accompanied by in vivo skeletal muscle mitochondrial dysfunction. Eur J Endocrinol 2008;158:643-53.

27. Nair KS, Bigelow ML, Asmann YW, Chow LS, CoenenSchimke JM, Klaus KA, et al. Asian Indians have enhanced skeletal muscle mitochondrial capacity to produce ATP in association with severe insulin resistance. Diabetes 2008;57: 1166-75.

28. Asmann YW, Stump CS, Short KR, Coenen-Schimke JM, Guo $\mathrm{Z}$, Bigelow ML, et al. Skeletal muscle mitochondrial functions, mitochondrial DNA copy numbers, and gene transcript profiles in type 2 diabetic and nondiabetic subjects at equal levels of low or high insulin and euglycemia. Diabetes 2006; 55:3309-19.

29. de Wilde J, Mohren R, van den Berg S, Boekschoten M, Dijk $\mathrm{KW}$, de Groot P, et al. Short-term high fat-feeding results in morphological and metabolic adaptations in the skeletal muscle of C57BL/6J mice. Physiol Genomics 2008;32:360-9.

30. Hancock CR, Han DH, Chen M, Terada S, Yasuda T, Wright DC, et al. High-fat diets cause insulin resistance despite an increase in muscle mitochondria. Proc Natl Acad Sci U S A 2008; 105:7815-20.

31. Turner N, Bruce CR, Beale SM, Hoehn KL, So T, Rolph MS, et al. Excess lipid availability increases mitochondrial fatty acid oxidative capacity in muscle: evidence against a role for reduced fatty acid oxidation in lipid-induced insulin resistance in rodents. Diabetes 2007;56:2085-92.

32. Garcia-Roves P, Huss JM, Han DH, Hancock CR, IglesiasGutierrez E, Chen M, et al. Raising plasma fatty acid concentration induces increased biogenesis of mitochondria in skeletal muscle. Proc Natl Acad Sci U S A 2007;104(25):10709-13.

33. Bajaj M, Medina-Navarro R, Suraamornkul S, Meyer C, DeFronzo RA, Mandarino LJ. Paradoxical changes in muscle gene expression in insulin-resistant subjects after sustained reduction in plasma free fatty acid concentration. Diabetes 2007;56:743-52.

34. Bonnard C, Durand A, Peyrol S, Chanseaume E, Chauvin MA, Morio B, et al. Mitochondrial dysfunction results from oxidative stress in the skeletal muscle of diet-induced insulin-resistant mice. J Clin Invest 2008;118:789-800.

35. Boon H, Blaak EE, Saris WH, Keizer HA, Wagenmakers AJ, van Loon LJ. Substrate source utilisation in long-term diagnosed type 2 diabetes patients at rest, and during exercise and subsequent recovery. Diabetologia 2007;50:103-12.

36. Larsen S, Ara I, Rabøl R, Andersen JL, Boushel R, Dela F, et al. Are substrate use during exercise and mitochondrial respiratory capacity decreased in arm and leg muscle in type 2 diabetes? Diabetologia 2009;52:1400-8.

37. Holloway GP, Thrush AB, Heigenhauser GJ, Tandon NN, Dyck DJ, Bonen A, et al. Skeletal muscle mitochondrial FAT/ CD36 content and palmitate oxidation are not decreased in obese women. Am J Physiol Endocrinol Metab 2007;292: E1782-9.

38. Holloway GP, Bonen A, Spriet LL. Regulation of skeletal muscle mitochondrial fatty acid metabolism in lean and obese individuals. Am J Clin Nutr 2009;89:455S-62S.

39. Desvergne B, Wahli W. Peroxisome proliferator-activated receptors: nuclear control of metabolism. Endocr Rev 1999;20: 649-88.

40. Finck BN, Bernal-Mizrachi C, Han DH, Coleman T, Sambandam N, LaRiviere LL, et al. A potential link between muscle peroxisome proliferator- activated receptor-alpha signaling and obesity-related diabetes. Cell Metab 2005;1:133-44.

41. Carling D. The AMP-activated protein kinase cascade--a unifying system for energy control. Trends Biochem Sci 2004;29: 18-24.

42. Randle PJ, GArland PB, Hales CN, Newsholme EA. The glucose fatty-acid cycle. Its role in insulin sensitivity and the metabolic disturbances of diabetes mellitus. Lancet 1963;1(7285): 785-9.

43. Koves TR, Ussher JR, Noland RC, Slentz D, Mosedale M, Ilkayeva $\mathrm{O}$, et al. Mitochondrial overload and incomplete fat- 
ty acid oxidation contribute to skeletal muscle insulin resistance. Cell Metab 2008;7:45-56.

44. Adams SH, Hoppel CL, Lok KH, Zhao L, Wong SW, Minkler $\mathrm{PE}$, et al. Plasma acylcarnitine profiles suggest incomplete long-chain fatty acid beta-oxidation and altered tricarboxylic acid cycle activity in type 2 diabetic African-American women. J Nutr 2009;139:1073-81.

45. Tilg $\mathrm{H}$, Moschen AR. Inflammatory mechanisms in the regulation of insulin resistance. Mol Med 2008;14:222-31.

46. Houstis N, Rosen ED, Lander ES. Reactive oxygen species have a causal role in multiple forms of insulin resistance. Nature 2006;440(7086):944-8.

47. Anderson EJ, Lustig ME, Boyle KE, Woodlief TL, Kane DA, Lin CT, et al. Mitochondrial $\mathrm{H} 2 \mathrm{O} 2$ emission and cellular redox state link excess fat intake to insulin resistance in both rodents and humans. J Clin Invest 2009;119:573-81.

48. Lee HY, Choi CS, Birkenfeld AL, Alves TC, Jornayvaz FR, Jurczak MJ, et al. Targeted expression of catalase to mitochondria prevents age-associated reductions in mitochondrial function and insulin resistance. Cell Metab 2010;12:668-74.

49. Barazzoni R, Zanetti M, Gortan Cappellari G, Semolic A, Boschelle M, Codarin E, et al. Fatty acids acutely enhance insulin-induced oxidative stress and cause insulin resistance by increasing mitochondrial reactive oxygen species (ROS) generation and nuclear factor- $\mathrm{kB}$ inhibitor (IKB)-nuclear factor- $\mathrm{KB}(\mathrm{NFkB})$ activation in rat muscle, in the absence of mitochondrial dysfunction. Diabetologia 2012;55:773-82.

50. Fisher-Wellman KH, Neufer PD. Linking mitochondrial bioenergetics to insulin resistance via redox biology. Trends Endocrinol Metab 2012;23:142-53. 